\title{
GRANULE CELL AS A SITE OF GENE ACTION IN THE WEAVER MOUSE CEREBELLUM: EVIDENCE FROM HETEROZYGOUS MUTANT CHIMERAS ${ }^{1}$
}

\author{
DAN GOLDOWITZ ${ }^{2}$ AND RICHARD J. MULLEN
}

Department of Anatomy, University of Utah School of Medicine, Salt Lake City, Utah 84132

Received February 4, 1982; Revised April 19, 1982; Accepted April 29, 1982

\begin{abstract}
Experimental mouse chimeras were used to determine the site(s) of gene action in the weaver mutant cerebellum. Chimeras containing mixtures of heterozygous weaver $(w v /+)$ and non-weaver $(+/+)$ cells were produced by the standard embryo aggregation technique. The non-weaver component of the chimera was chosen so that Purkinje cells or granule cells could be distinguished histologically from weaver Purkinje or granule cells. Levels of $\beta$-glucuronidase activity were used to mark Purkinje cells, with the weaver strain having a high $\beta$-glucuronidase activity, while the nonweaver strain had low $\beta$-glucuronidase activity. The increased centralized clumping of heterochromatin in ichthyosis (ic) mutant mice compared to non-ic mice was used to mark granule cell populations. In the weaver chimera, there was a decreased cerebellar size, decreased numbers of Purkinje and granule cells, and increased ectopic Purkinje and granule cells compared to nonweaver, control mice. With the glucuronidase cell marker, it was found that there was no correlation between ectopia and genotype; that is, genetically normal cells, as well as weaver cells, were found in ectopic positions. Thus, the weaver gene acts extrinsic to the Purkinje cells in creating the ectopia characteristic of heterozygous weaver mutants. Analysis of the ectopic granule cells, however, revealed that $100 \%$ of the ectopic granule cells were from the weaver component of the chimera. Thus, the weaver gene intrinsically affects granule cells in causing ectopia. Other hypothetical sites of gene action would produce a genetically mixed population of ectopic granule cells, which was not the case in this study. These findings are discussed in relation to other abnormalities in the heterozygous weaver mutant and in regard to the Bergmann glia and homozygous mutant. Finally, speculations on the nature of the granule cell deficit are discussed briefly.
\end{abstract}

Genetic mutations which affect brain cytoarchitectonics have proven very useful in the study of events that are critical to the development of brain structures (SidInan, 1968; Caviness and Rakic, 1978; Sotelo, 1980). Analyses of heterozygous and homozygous weaver $(w v) \mathrm{mu}-$ tant mice have led to several important observations on cerebellar development, such as: influences on dendritic structure (Rakic and Sidman, 1973c; Sotelo, 1975; Hirano

\footnotetext{
1 These studies were initiated while we were associated with the Department of Neuropathology, Harvard Medical School and the Department of Neuroscience, Children's Hospital Medical Center, Boston. This work was supported by grants to R. J. M. from the National Institutes of Health (Grant NS 16156) and the March of Dimes Birth Defects Foundation (Basic Research Grant 1-684). We thank Anne Moody Mjaatvedt for her expert technical assistance.

${ }^{2}$ To whom correspondence should be addressed at his present address: Department of Anatomy, 'Thomas Jefferson University, 1020 Locust Street, Philadelphia, PA 19107.
}

and Dembitzer, 1973; Hirano et al., 1977), neuronal interactions in the formation of synaptic contacts (Sotelo, 1975), the impetus for foliation (Rakic and Sidman, 1973c), and the role of Bergmann glial cells and their fibers in guiding migrating granule cells (Rakic and Sidman, 1973a). In order to extend these observations to explanations of cellular interactions during development, it is necessary to know to what extent the observed pathology is related to the primary effects of the mutation. Thus, a true picture of the events that mold the weaver cerebellum can be achieved only by knowing the site(s) of gene action.

The most noticeable deficit in the homozygous weaver cerebellum is its diminutive size. This is largely due to the nearly complete absence of granule cells, the most numerous neuron in the normal cerebellum. In addition to this marked loss of granule cells, there are also deficits in the number of Purkinje cells (Rezai and Yoon, 1972) and Bergmann glia cells (also known as Golgi epithelial 
cells; Rakic and Sidman, 1973a). Many of the Purkinje and Bergmann glia cells that remain are ectopic and have misshapen apical processes. The cerebellum of heterozygous weaver mice $(w v /+)$, which have only a single dose of the weaver gene, is affected in the same fashion as that of $w v / w v$ mice but with a greatly attenuated pathology (Rezai and Yoon, 1972; Rakic and Sidman, 1973b).

The granule cell deficits in the heterozygous and homozygous weaver cerebellum have been attributed to a disorder of the Bergmann glia cells (Rakic and Sidman, 1973a). Observations of the developing cerebellum indicated that the granule cells are closely apposed to Bergmann glia fibers during the course of their migration from the external to the internal granular layer (Mugnaini and Forstrønen, 1967; Rakic, 1971). Based upon an analysis of granule cell production and migration in $w v /+$ cerebellum, Rezai and Yoon (1972) concluded that the granule cell deficit was a result of faulty migration. The observation of aberrant Bergmann glia fibers and reduced numbers of Bergmann glia cells in $w v /+$ and $w v / w v$ cerebella provided evidence to argue persuasively the case for a vital role in granule cell migration for the radially oriented Bergmann glia (Rakic and Sidman, $1973 \mathrm{a}, \mathrm{b}, \mathrm{c})$. A subsequent finding that Bergmann glia cells are present in the prenatal mouse cerebellum (Swarz and Oster-Granite, 1978) provided the necessary temporal sequencing for an important role for glia in guiding the successful migration of cerebellar granule cells. Evidence contrary to this view came from other studies of the weaver cerebellum. Bignami and Dahl (1974) used the immunofluorescent localization of glial fibrillary acidic protein to show that Bergmann glia fibers were normal in $w v /+$ mice and not as severely affected in $w v / w v$ mice as had been reported originally by Rakic and Sidman (1973a). Furthermore, ultrastructural observations of the $w v / w v$ cerebellum showed that a few granule cells were able to migrate through the molecular layer into the internal granule layer where they then died, suggesting that the defect in migration was not the cause of granule cell fatality (Sotelo and Changeux, 1974). These ultrastructural studies by Sotelo and Changeux (1974) supported the immunofluorescent findings concerning the presence of Bergmann glia in the weaver cerebellum. From this work, Sotelo and Changeux suggested that the granule cells "are closer than the glial cells" to the primary target of the mutation. The cause of the Purkinje cell abnormalities in this mutation, however, has not been investigated.

It is conceivable that the mutant gene may be acting in any, all, or none of the affected cell types. Experimental mouse chimeras have been used successfully to determine the site of gene action in other cerebellar mutants (see Mullen and Herrup, 1979). By this method, an 8-cell embryo of the mutant genotype is aggregated with an 8cell embryo of the wild type genotype $(+/+)$ to produce a mouse with cells derived from both genotypes. The choice of the $+/+$ embryo is based upon a particular genetic cell marker which will differentiale $+/+$ cells from the mutant cells in the chimera. By analyzing the genotype of cells (via cell markers) compared to the phenotype (normal or mutant), one can implicate directly or rule out a particular cell type as a site of the mutant gene's action.

In this study, we examined $w v /-^{3}$ chimeras to determine if the cerebellar pathology of the $w v /+$ mouse could be attributed to the weaver gene acting in both or either of the primarily affected neuronal cell types: the Purkinje and/or the granule cell. A cell-marking system for the Bergmann glia cell has not yet been established in our laboratory, and therefore, in this study, we were unable to assess the weaver gene's action upon this cell type.

$\beta$-Glucuronidase was used as the marker for Purkinje cells (Mullen, 1977a). Most weaver mice in our colony are homozygous for the structural gene that determines high levels of $\beta$-glucuronidase activity $\left(G u s^{b} / G u s^{b}\right)$. Embryos from a low $\beta$-glucuronidase strain $\left(G u s^{h} / G u s^{h}\right)$ of mice were used as the other component of the chimera. Ichthyosis (ic/ic) mice have an abnormal, central accumulation of heterochromatin in the nucleus of cerebellar granule cells (Goldowitz and Mullen, 1982), and this nuclear phenotype was used to identify $w v /-+/+$ granule cells from $+/+i c / i c$ granule cells in experimental weaver $\leftrightarrow$ ichthyosis chimeras. In this paper, we report on our analyses of $w v /-G u s^{b} / G u s^{b} \leftrightarrow+/+G u s^{h} / G u s^{h}$ and $w v /-+/+\leftrightarrow+/+i c / i c$ chimeras. These studies indicate that the weaver locus acts extrinsic to the Purkinje cells but intriinsic to the granule cells in producing the heterozygous weaver cerebellum.

\section{Materials and Methods}

Mice and chimera production. Five strains of mice that carried the weaver mutation were maintained in our laboratory for use in these experiments. The genetic backgrounds of these strains were as follows: $\mathrm{C} 57 \mathrm{BL} / 6$ $b g / b g$ (beige); B6CBA (obtained from the Jackson Laboratory, Bar Harbor, ME); $\mathrm{B} 6 \mathrm{C} 3 \mathrm{H} b g / b g$ (beige); a $\mathrm{B} 6 \mathrm{C} 3 \mathrm{H}$-derived strain that was heterozygous at the beige, $\beta$-glucuronidase, and agouti loci; and this same strain carrying the ichthyosis gene. Chimeras were made as described by Mullen and Whitten (1971). In many of the experiments, ovulation was induced by the use of 1 to 2 IU of pregnant mare serum (Sigma, St. Louis, MO) followed in $44 \mathrm{hr}$ by the same dose of human chorionic gonadotropin (Sigma). Two days after the detection of a vaginal plug, the female's oviducts were flushed to harvest the 8-cell embryos. The zona pellucida was removed from the embryos by gentle Pronase treatment. Embryos were aggregated and cultured overnight. Fused embryos that had reached the late morula or early blastula stage of development were transferred to the uteri of pseudopregnant $\mathrm{B} 6 \mathrm{D} 2 \mathrm{~F}_{1}$ mice.

For the production of chimeras designed to study the Purkinje cells, $\beta$-glucuronidase was used as the indepen-

\footnotetext{
${ }^{3}$ Because there is no linked marker for the weaver mutation and since heterozygous matings were used to produce weaver embryos, we could not verify with assurance that the weaver chimeras analyzed carried a single $(w v /+)$ or double $(w v / w v)$ dose of the weaver gene. Therefore, in referring to the weaver chimeras, we use the symbol $w v /-$ to insert a note of caution in assigning genotype. For reasons that will be apparent under "Results," the $w v /$ - symbol probably could be interchanged with $w v /+$, but this point will be addressed under "Discussion."
} 
dent cell marker to distinguish between weaver and normal genotypes. The weaver embryos had the Gus allele determining high $\beta$-glucuronidase activity $(w v /+$ $\left.G u s^{b} / G u s^{b}\right)$. The low $\beta$-glucuronidase $\left(G u s^{h} / G u s^{h}\right)$ component of the chimera was derived from matings of $\mathrm{C} 3 \mathrm{H}$ and CBA or CBA and CBA mice $\left(+/+G u s^{h} / G u s^{h}\right)$. For the production of chimeras designed to study the granule cells, the ichthyosis (ic) nuclear phenotype was used as the differential cell marker (see Goldowitz and Mullen, 1982). Weaver embryos $+/+$ at the ichthyosis (ic) locus were used $(w v /++/+)$. Homozygous ichthyosis embryos $+/+$ at the $w v$ at locus were used for the other component of the chimera $(+/+i c / i c)$. Two $w v /-G u s^{b} / G u s s^{b} \leftrightarrow$ $+/+G u s^{h} / G u s^{h}$ and eight $w v /-+/+\leftrightarrow+/+i c / i c$ chimeras were analyzed for this study.

Marking of Purkinje cells with $\beta$-glucuronidase histochemistry. Mice were perfused with a $4 \%$ paraformaldehyde solution in $0.1 \mathrm{M}$ phosphate buffer ( $\mathrm{pH} 7.3$ ) at $4^{\circ} \mathrm{C}$. Whole heads were immersed in the $4^{\circ} \mathrm{C}$ perfusate solution for 2 to $4 \mathrm{hr}$. The brains then were removed and postfixed for an additional 2 to $4 \mathrm{hr}$ in the same cold perfusion solution. The brains were rinsed with daily changes of a $10 \%$ polyvinylpyrrolidone (Sigma), $6 \%$ sucrose solution for 4 days. This was followed by a single change of cold water and three changes of cold $98 \%$ acetone. Brains were infiltrated with polyethylene glycol 400 distearate (Ruger Chemical, Irvington, NJ) and embedded in the same medium. Sagittal, $8-\mu \mathrm{m}$ sections were cut on a microtome and stained for the histochemical demonstration of $\beta$-glucuronidase according to the procedure of Hayashi et al. (1964) as modified by Feder (1976). Sections were stained three times, with the addition of new media each time, for a total period of 10 to 72 hr. The reaction product was red and brain sections were counterstained lightly with methyl green. Purkinje cells with just the green counterstain were identified as belonging to the Gus $/ G u s^{h}$ genotype, whereas Purkinje cells with deposits of red stain were identified as being derived from the $G u s^{b} / G u s^{b}$ genotype. Two $G u s^{b} / G u s^{b}$

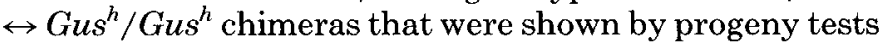
to be $+/+$ at the weaver locus were used as age-matched controls. Two $w v /+$ mice were analyzed to quantify the heterozygous weaver effect upon Purkinje cell numbers, the length of the Purkinje cell layer, and Purkinje cell ectopia (consisting of clearly identifiable Purkinje cells removed by at least one somal diameter from the row of aligned Purkinje cells). These measurements were made from sections that were approximately $880 \mu \mathrm{m}$ lateral to midline (according to Sidman et al., 1971). This region of the cerebellum is characterized by the most medial attachment site between the cerebellum and brainstem and the lateralmost portion of the lingula.

Marking granule cells using the ichthyosis (IC) nuclear phenolype. Mice were perfused with one-fourth and then one-half strength Karnovsky's fixative in $0.1 \mathrm{M}$ phosphate buffer, pH 7.2 (Karnovsky, 1965). Brains were removed from the skull after $1 \mathrm{hr}$ of postfixation and stored in one-half strength fixative overnight. On the following day, each side of the cerebellum was cut in four or five sagittal slices. Tissue was placed in a $2 \%$ osmium tetroxide, $5 \%$ sucrose, $0.1 \mathrm{~m}$ phosphate-buffered solution for $90 \mathrm{~min}$; rinsed with water and acetate buffer; and stained overnight with a slightly acidic solution of $0.5 \%$ uranyl acetate. Tissue slices then were embedded in either Epon or an Epon/Araldite mixture. Toluidine blue was used to stain $1-\mu \mathrm{m}$ sections cut on an LKB V Ultrotome. The IC nuclear phenotype, consisting of large, clumped, centrally placed masses of heterochromatin, was readily evident in $1-\mu \mathrm{m}$ sections stained with toluidine blue. As previously determined (Goldowitz and Mullen, 1982), a granule cell was graded as IC positive if the area of the centrally placed mass of heterochromatin equaled or exceeded $10 \%$ of the total area of the nucleus. In our initial work, measurements were made from photomicrographs (magnification $\times 2,460$ ) with a Numonics area analyzer. In subsequent work, granule cells were measured directly from the microscope with a drawing tube attachment (final magnification $\times 600$ ). The person scoring the cells was unaware of the source of the tissue. Questionable cells were traced and measured with the Numonics area analyzer to determine whether the cell was IC positive or negative. A population of at least 300 granule cells from the granular layer of the anteromedial cerebellum was analyzed to determine the expression of the IC phenotype in granule cells residing in the granular layer. The expression of the IC phenotype in ectopic granule cells residing in the molecular layer was determined from one complete $1-\mu \mathrm{m}$ section of the cerebellum or a region large enough to have about 300 such ectopic granule cells. Thus, the expression of the IC nuclear phenotype was determined for granule cells in the granular and molecular layers of five groups of mice: (1) $+/++/+$ mice (i.e., $+/+$ at the weaver and ichthyosis loci), (2) $w v /++/+$ mice, $(3)+/+i c / i c,(4) w v /+i c / i c$, and (5) chimeras that were $w v /-+/+\leftrightarrow+/+i c / i c$. The number of granule cells in single $1-\mu \mathrm{m}$ midsagittal sections was estimated by multiplying the granule cell density (sample area of approximately $0.05 \mathrm{~mm}^{2}$ ) by the total area of the granular layer.

Determination of degree of chimerism. The chimerism of Purkinje and granule cells was determined quantitatively. In $w v /-G u s^{b} / G u s^{b} \leftrightarrow+/+G u s^{h} / G u s^{h}$ chimeras, cach Purkinje cell in a population of between 400 and 700 was scored as being derived from either the $G u s^{b}$ component (red stain in cells) or the $G u s^{h}$ component (no red stain). From these data, the proportion of mutant and normal Purkinje cells in the chimera was determined.

The degree of chimerism of a granule cell population was determined from the following equation:

\begin{tabular}{lcc}
$\begin{array}{l}\% \text { IC-positive cells in } \\
\text { chimera }\end{array}$ & - & $\begin{array}{c}\text { mean } \% \text { IC-positive cells } \\
\text { in }+/+ \text { mice }\end{array}$ \\
\hline $\begin{array}{l}\text { mean \% IC-positive cells } \\
\text { in } i c / \text { ic mice }\end{array}$ & - & $\begin{array}{c}\text { mean } \% \text { of IC-positive cells } \\
\text { in }+/+ \text { mice }\end{array}$
\end{tabular}

The resulting fraction gives the calculated proportion of cells in the population of granule cells analyzed that are derived from the ichthyosis component (i.e., non-weaver) of the chimera.

\section{Results}

Two $w v /-G u s^{b} / G u s^{b} \leftrightarrow+/+G u s^{h} / G u s^{h}$ chimeras (A and $B$ ) were produced and used to analyze the site of action of the $w v$ gene relative to the Purkinje cells. Eight $w v /-+/+\leftrightarrow+/+i c / i c$ chimeras (C through $\mathrm{J}$ ) were produced and used to analyze the site of action of the $w v$ gene relative to the granule cells. All but two of the 
chimeras $(\mathrm{H}$ and $\mathrm{J})$ had coats that indicated a mixture of both genotypes (beige and agouti for $w v /-G u s^{b} / G u s^{b}$ $a / a b g / b g \leftrightarrow+/+G u s^{h} / G u s^{h} \mathrm{~A} / \mathrm{A}+/+$ and hair and no hair for $w v /-+/+\leftrightarrow+/+i c / i c)$. Chimera $\mathrm{H}$ appeared to be all $i c / i c$, and chimera J was all $w v /-$. In the $w v /-$ $G_{u s}^{b} / G^{b} s^{b} \leftrightarrow+/+G u s^{h} / G^{h} s^{h}$ chimeras, $59 \%$ and $65 \%$ of the Purkinje cells stained positively for high $\beta$-glucuronidase activity. In the $w v-+/+\leftrightarrow+/+i c / i c$ chimeras, varying proportions of the granular layer granule cells were calculated to be derived from the $w v /-$ component (see Table V). As can be seen from the percentages in Table V, chimeras $\mathrm{C}$ through $\mathrm{G}$ displayed an internal granular layer with granule cells from both the $i c$ and $w v /-$ genotypes. It was calculated that, in chimera $\mathbf{H}$, $100 \%$ of the granule cells in the internal granule cell layer were $i c / i c$ in genotype, whereas in chimeras I and J, 95\% and $100 \%$ were $w v /+$ in genotype. Thus, these three chimeras did not exhibit mosaicism in the portions of the cerebellum examined. Several features of the seven $w v /$ $-\leftrightarrow+/+$ chimeric cerebella (A through G and I) indicated that both the $w v /-$ and $+/+$ genotypes were being expressed. The sizes of the cerebellum of these chimeras were smaller than $+/+$ controls but larger than $w v /+$ cerebella (Fig. 1; Table I). This was due to two features of the weaver cerebellum that are expressed in $w v /-\leftrightarrow$ $+/+$ cerebella: (1) a decreased number of granule cells in the internal granule cell layer and (2) a decreased number of Purkinje cells. These deficits are quantified in Table II. Because of the reduction in granule and Purkinje cell populations that normally occurs in $w v /+$ cerebellum, the real proportion of the weaver component in the chimeras might be higher than the percentages noted if there is a selective reduction in cell populations derived from the $w v /-$ component.

The most apparent expression of the $w \dot{v} /-$ component in chimeric cerebella is the deviation from the strict segregation of cellular populations that occurs in normal cerebella. The granule cell layer, Purkinje cell layer, and molecular layer, which are obviously delineated in the $+/+$ cerebellum (Figs. $2 a$ and $3 a$ ), are less distinct in $w v /+$ (Figs. $2 b$ and $3 b$ ) and some $w v /-\leftrightarrow+/+$ cerebella (Figs. 2, $c$ and $d$, and 3, $d$ and $e$ ). This is because there is a much more frequent occurrence of granule, Purkinje, and apparent Bergmann glia cell ectopia in the cerebellum of $w v /+$ and $w v /-\leftrightarrow+/+$ mice. In particular, Purkinje cells are found in the granule cell and molecular layers and granule cells are found in the molecular layer. Many cells that had the $\beta$-glucuronidase staining characteristics of Bergmann glia were seen displaced to the molecular layer in $w v /+$ and $w v /-\leftrightarrow+/+$ cerebella (Fig. $2, b$ and $c$ ). This appears to be an additional feature in the general disarray of the weaver cerebellum. The numbers of ectopic Purkinje and granule cells in $+/+, w v /+$, and $w v /-\leftrightarrow+/+$ cerebella are shown in Table III. Chimeras $\mathrm{A}$ through $\mathrm{F}$ have an intermediate expression of the $w v /+$ phenotype of displaced cells. Chimeras $\mathrm{G}$ and $\mathrm{H}$ and $\mathrm{I}$ and $\mathrm{J}$ show an expression of phenotype that is predominantly $i c / i c$ and $w v /+$, respectively. Thus, such features of the $w v /+$ cerebellum as the reduction in the number of Purkinje and granule cells, the ectopia of granule, Purkinje, and Bergmann glia cells, and the reduced cerebellar size all were expressed in $w v /-\leftrightarrow+/+$ cerebella.
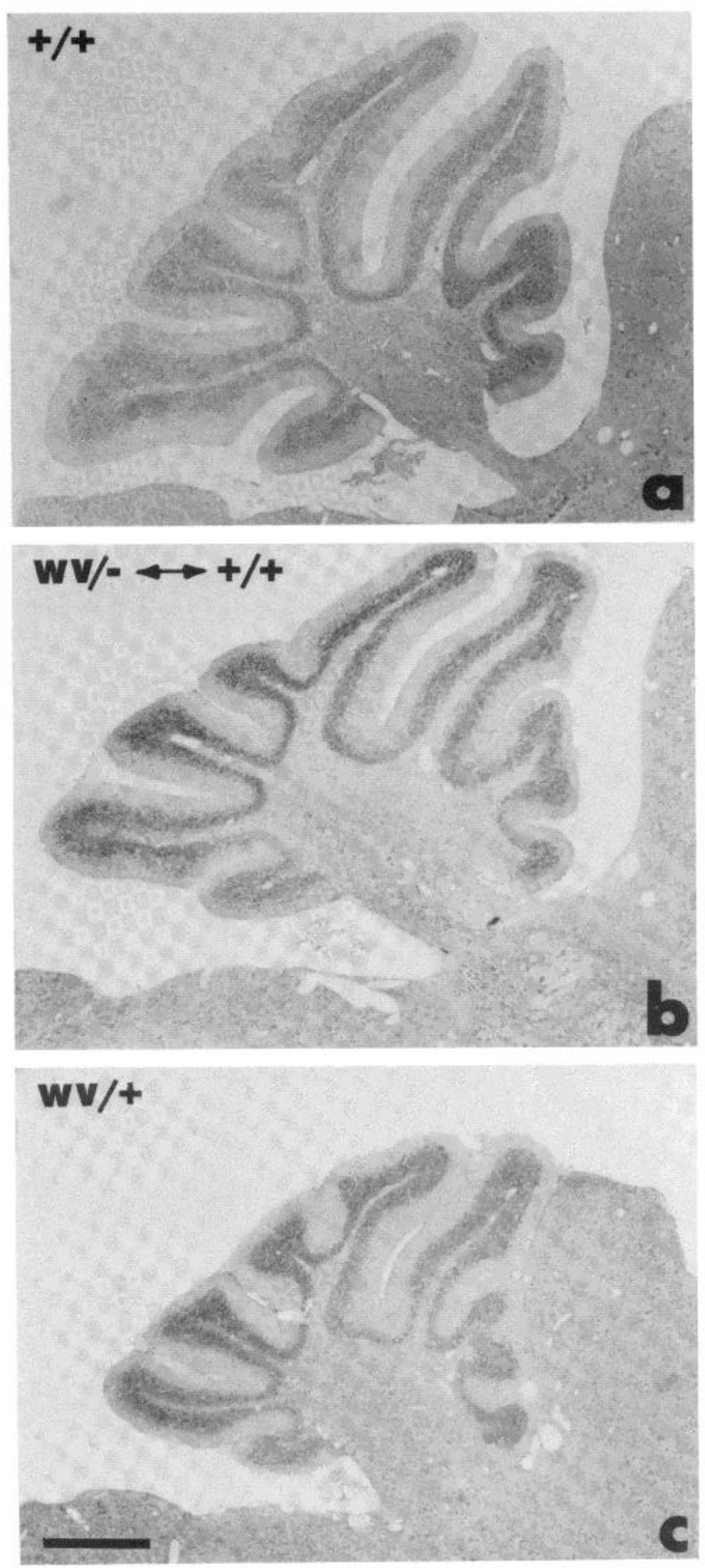

Figure 1. Photomicrographs at low magnification of sagittal sections of whole cerebella from $+/+, w v /-\leftrightarrow+/+$, and $w v /+$ mice. $a$, The cerebellum from a wild type $(+/+)$ mouse shows a richly populated granule cell region inferior to the 1cell-thick Purkinje cell layer. The molecular layer is basically free of granule, Bergmann glia, and Purkinje cells. $b$, The cerebellum of a $w v /-\leftrightarrow+/+$ chimera from a similar sagittal plane of section shows attenuated characteristics of the heterozygous weaver cerebellum $(c)$ : namely, a reduced cerebellar size, a thinner, less dense granule cell layer, and a less strict alignment of cell types due to ectopic granule cells and misaligned Purkinje and Bergmann glia cells. Cerebella were wax embedded, sectioned at $8 \mu \mathrm{m}$, and stained for $\beta$-glucuronidase activity. In $a$ through $c$, the calibration bar $=250 \mu \mathrm{m}$. 
TABLE I

Size of cerebellum as measured by the length of the Purkinje cell layer

The difference in the absolute values among the animals in each group is due to the greater shrinkage of tissue processed for wax as compared to plastic embedding and the more lateral plane of section used to analyze the wax-embedded tissue compared to the medial plane of section used to analyze plastic-embedded tissue. Therefore, only relative comparisons can be made between the data in the two groups.

\begin{tabular}{ccc}
\hline Genotype & Size & $\bar{X}$ \\
\hline
\end{tabular}

Wax embedded ${ }^{a}$

Controls

$\mathrm{Gus}^{b} / \mathrm{Gus}^{b} \leftrightarrow \mathrm{Gus}^{h} / \mathrm{Gus}^{h}$

(non-weaver chimeras) $\quad 14.9$

$w v /+\quad 12.0$

10.8

Chimeras

$w v /-G u s^{b} / G u s^{b} \leftrightarrow+/+G u s^{h} / G u s^{h}$

A

12.4

B

\begin{tabular}{lll}
$\begin{array}{l}\text { Epon embedded } \\
\text { Controls } \\
+/++/+\end{array}$ & & \\
& & \\
& & \\
& & \\
$+/+i c / i c$ & 25.6 & $24.0 \pm 1.0$ \\
& 22.2 & \\
& 24.8 & \\
& 26.5 & $24.9 \pm 0.6$ \\
$w v /++/+$ & 23.7 & \\
& 24.6 & \\
$w v /+i c / i c$ & 22.1 & $21.3 \pm 0.4$ \\
& 20.9 & \\
Chimeras & 21.0 & \\
$w v /-+/+\leftrightarrow+/+i c / i c$ & & \\
$\mathrm{C}$ & & \\
$\mathrm{D}$ & & \\
$\mathrm{E}$ & 23.6 & $23.2 \pm 0.4$ \\
$\mathrm{~F}$ & 23.3 & \\
$\mathrm{G}$ & 23.7 & \\
$\mathrm{H}$ & 23.3 & \\
$\mathrm{I}$ & 23.4 & \\
$\mathrm{~J}$ & 25.2 & \\
\hline
\end{tabular}

${ }^{a}$ Wax-embedded material ( $8-\mu \mathrm{m}$ sections) from a region about 900 $\mu \mathrm{m}$ lateral to the midsagittal plane.

${ }^{b}$ Epon-embedded material ( $1-\mu \mathrm{m}$ sections) from the midsagittal plane.

Purkinje cell analysis. The position of Purkinje cells situated in ectopic positions (at least one Purkinje cell diameter from the Purkinje cell layer) was recorded. Purkinje cells in the molecular layer could be easily identified based upon the size of the cell body. The identification of ectopic Purkinje cells in the granule cell layer required more certainty because another group of relatively large neurons, the Golgi neurons, also occur in this region. In a particular plane of section, the defining characteristics of the Purkinje or Golgi neuron could be masked. To avoid mistakes in classifying ectopic cells, questionable cells were differentiated positively as either Purkinje or Golgi cells by examining the sections of cerebellum that immediately followed and preceded the cells in question. A rough, three-dimensional reconstruction from these serial sections of the questionable cells made it clear if the cell was of the Purkinje or Golgi type. Each verified Purkinje cell then was examined to determine if deposits of red stain were present (a $u v /-$ $G u s^{b} / G u s^{b}$ cell) or absent (a $+/+G u s^{h} / G u s^{h}$ cell). The ectopic Purkinje cells then were divided into two groups: those that arose from the $w v /-G u s^{b} / G u s^{b}$ genotype and those that arose from the $+/+G u s^{h} / G u s^{h}$ genotype. As Table IV and Figure 2 show, there were ectopic Purkinje cells derived from each component in a proportion similar to that calculated for the entire Purkinje cell population.

Granule cell analysis. In five of the eight $w v /-+/+$ $\leftrightarrow+/+i c / i c$ cerebella, the observed expression of the IC phenotype in the granule cells of the granular layer was different from $+/+, w v /+$, or $i c / i c$ cerebella (Fig. 3; Table

TABLE II

Expression of neuronal deficits in $w v /-\leftrightarrow+/+$ chimeric mouse cerebellum

\begin{tabular}{|c|c|c|}
\hline Purkinje Cells & No./Section ${ }^{a}$ & $\bar{X}$ \\
\hline \multicolumn{3}{|l|}{ Controls } \\
\hline \multirow{2}{*}{$\begin{array}{r}G u s^{b} / G u s^{b} \leftrightarrow G u s^{h} / G u s^{h} \\
\text { (non-weaver chimeras) }\end{array}$} & 428 & 459.5 \\
\hline & 491 & \\
\hline \multirow[t]{2}{*}{$w v /+$} & 372 & 361.5 \\
\hline & 351 & \\
\hline \\
\hline \multirow{2}{*}{\multicolumn{2}{|c|}{$\begin{array}{l}w v /-G u s^{b} / G u s^{b} \leftrightarrow+/+G u s^{h} / G u s^{h} \\
\text { A }\end{array}$}} & \\
\hline & 358 & 388.5 \\
\hline $\mathrm{B}$ & 419 & \\
\hline Granule Cells & No./Section ${ }^{h}$ & $\bar{X} \pm$ SEM \\
\hline \multicolumn{3}{|l|}{ Controls } \\
\hline \multirow[t]{3}{*}{$+/++/+$} & 32,247 & $28,793 \pm 2,814$ \\
\hline & 30,915 & \\
\hline & $23,217^{\circ}$ & \\
\hline \multirow[t]{4}{*}{$+/+i c / i c$} & 35,431 & $35,278 \pm 1,545$ \\
\hline & 34,495 & \\
\hline & 39,322 & \\
\hline & $31,865^{c}$ & \\
\hline \multirow[t]{3}{*}{$w v /++/+$} & 22,324 & \\
\hline & 19,523 & $19,564 \pm 1,582$ \\
\hline & $16,845^{c}$ & \\
\hline
\end{tabular}

Weaver chimeras

$\begin{array}{lll}w v / \cdots+/+\leftrightarrow+/+i c / i c & \\ \mathrm{C} & 25,464 & 20,981 \pm 1,143 \\ \mathrm{D} & 19,438 & \\ \mathrm{E} & 21,345 & \\ \mathrm{~F} & 19,709 \\ \mathrm{G} & 21,840 \\ \mathrm{H} & 25,425 \\ \mathrm{I} & 16,450 \\ \mathrm{~J} & 18,175\end{array}$

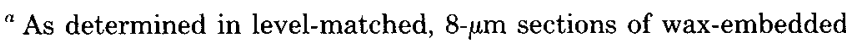
cerebella; each value is the average of counts from two cerebellar sections.

${ }^{b}$ Estimated the total number of granule cells in a $1-\mu \mathrm{m}$, midsagittal cerebellar section as determined from the total area occupied by the granular layer multiplied by the density of granule cells.

'From a group of three animals processed at the same time in the usual manner. For unknown reasons, all had smaller than normal granular cell areas. 
TABLE III

Expression of neuronal ectopia in wv/ $\leftrightarrow+/+$ chimeric mouse cerebelleum

\begin{tabular}{lcc}
\hline \multicolumn{1}{c}{ Purkinje Cells } & No./Section $^{a}$ & $\bar{X}$ \\
\hline Controls & & \\
$G u s^{b} / G u s^{b} \leftrightarrow G u s^{h} / G u s^{h}$ & 1.0 & \\
(non-weaver chimeras) & 1.5 & 1.2 \\
$w v /+$ & 34.5 & \\
& 55 & 44.8
\end{tabular}

Chimeras $w v /-G u s^{b} / G u s^{b} \leftrightarrow+/+G u s^{h} / G u s^{h}$ B

\section{9}

17.0

\begin{tabular}{lcc}
\hline \multicolumn{1}{c}{ Granule Cells } & $\begin{array}{c}\text { No./mm of } \\
\text { Purkinje } \\
\text { Cell Layer }^{b}\end{array}$ & $\tilde{X}$ \\
\hline Controls & & \\
$+/++/+$ & 7.4 & 5.9 \\
& 7.3 & \\
$+/+i c / i c$ & 3.1 & \\
& 5.1 & 2.9 \\
& 2.3 & \\
$w v /++/+$ & 1.6 & \\
& 2.6 & \\
$w v /+i c / i c$ & 36.8 & 36.8 \\
& 32.6 & \\
Chimeras & 41.0 & \\
$w v /-+/+\leftrightarrow+/+i c / i c$ & 39.9 & \\
C & & \\
D & & \\
E & & \\
F & 31.8 \\
G & 30.9 & 20.9 \\
H & 116 & \\
J & 22.8 \\
\hline
\end{tabular}

"Each measure is an average of two counts from complete cerebellar sections from the midsagittal plane; $8-\mu \mathrm{m}$ wax-embedded tissue was used.

${ }^{b}$ Only granule cells that were clearly removed from the main Purkinje cell layer were counted. In $w v /+$ and $w v /-$ chimeras, where the Purkinje cell layer was in some disarray, many ectopic granule cells were not counted because of their relationship to ectopic Purkinje cells. Thus, the values presented should be considered as conservative estimates. Sampling procedures are described under "Materials and Methods."

V). The percentages of ichthyosis-positive granule cells in the granular layer (those cells having $10 \%$ or more of their nuclear area occupied by centrally clumped heterochromatin) in chimeras $\mathrm{C}$ to $\mathrm{G}$ were $14.9,11.2,11.1,15.4$, and 17.2. These compare to a mean positive score of $26.7 \%$ for $i c / i c$ mice and $8.3 \%$ and $5.8 \%$ for $w v /+$ and $+/+$ mice, respectively. In the five chimeras, the calculated percentage of granular layer granule cells derived from the $w v /$ - was $56,74,75,54$, and 45 . In chimeras $\mathrm{H}$, and I and $J$, the percentages of ichthyosis-positive granule cells in the granular layer closely corresponded with expected values for the $i c / i c$ and $w v /+$ control cerebellum, respectively.
In order to determine the genotype of the granulc cells that failed to migrate and remained in the molecular layer, the same analysis was performed on ectopic granule cells. Granule cells in the molecular layer could be easily distinguished from other cells by the color of the nucleoplasm, the paucity of cytoplasm, and their small size. There is a very small population of ectopic granule cells residing in the molecular layer of $+/+$ mice (see Landis, 1973). This is also the case for $i c / i c$ mice. The numbers of ectopic granule cells in some $w v /-+/+\leftrightarrow$ $+/+i c / i c$ cerebella were more than in control mice but less than in $w v /+$ cerebella (Fig. 3; Table III). The expression of the IC phenotype in the ectopic granule cells of $i c / i c$ and $w v /+$ mice was similar to that found for granule cells in the granular layer for each genotype: low levels for $w v /+$ cerebella and much higher levels for ic/ic cerebella (Fig. 3; Table V). This was not the case for four of the $w v /-+/+\leftrightarrow+/+i c / i c$ chimeras (C to F). Only $3.3 \%, 5.0 \%, 2.3 \%$, and $5.1 \%$ of the ectopic granule cells expressed the IC phenotype. These percentages are what should be obtained for nonichthyosis cerebella and are in sharp contrast to the $14.9 \%, 11.2 \%, 11.1 \%$, and $15.4 \%$ of the granular layer granule cells which were positive for the IC phenotype in the same chimeras (see Table V). The calculated degree of chimerism of the molecular layer granule cells in each of these chimeras is 0 . That is, $100 \%$ of the cells in these four chimeras are calculated to have arisen from the $w v /$ - genotype and $0 \%$ from the ic/ic genotype. In contrast to the ectopic Purkinje cell population, the ectopic granule cells are derived only from the $w v /-$ genotype and do not at all reflect the general chimerism of the cerebellum (i.e., size, granule cell number, and total populations of ectopic granule cells). The number of ectopic granule cells (Table III) in chimeras $G$ and $H$ and $I$ and $J$ indicates the predominant participation of ichthyosis (with few ectopic cells) and weaver (with larger numbers of ectopic cells), respectively, in the granule cell colonization of the cerebellum.

The fact that few ectopic granule cells in chimeras $\mathrm{C}$ to F were IC in phenotype was not due to the inability of ectopic cells to express the phenotype or to any interaction between the $w v$ and ic loci since: (1) the small, normal population of ectopic granule cells in the $i c / i c$ cerebellum express the IC phenotype (Fig. $3 a$ ) and (2) in the $w v /+i c / i c$ double mutant cerebellum, the larger population of ectopic granule cells expressed the IC phe-

\section{TABLE IV}

Genotype of normally positioned and ectopic Purkinje cells expressed as a ratio of $G_{u s}^{b}$ and $G u s^{h}$ cells in $w v /+G u s^{b} / G u s^{b} \leftrightarrow$ $+/+$ Gus $^{h} /$ Gus $^{h}$ mice

Ratios are based on the total counts of two $8-\mu \mathrm{m}$ sections of cerebellum from similar sagittal planes of section.

\begin{tabular}{lc}
\hline Purkinje Cells & $G u s^{b} / G^{h} s^{h}: G u s^{h} / G u s^{h}$ \\
\hline Normally positioned & \\
Chimera A & $59: 41$ \\
Chimera B & $65: 35$ \\
& \\
Ectopic & \\
Chimera A & $58: 42$ \\
Chimera B & $53: 47$ \\
\hline
\end{tabular}



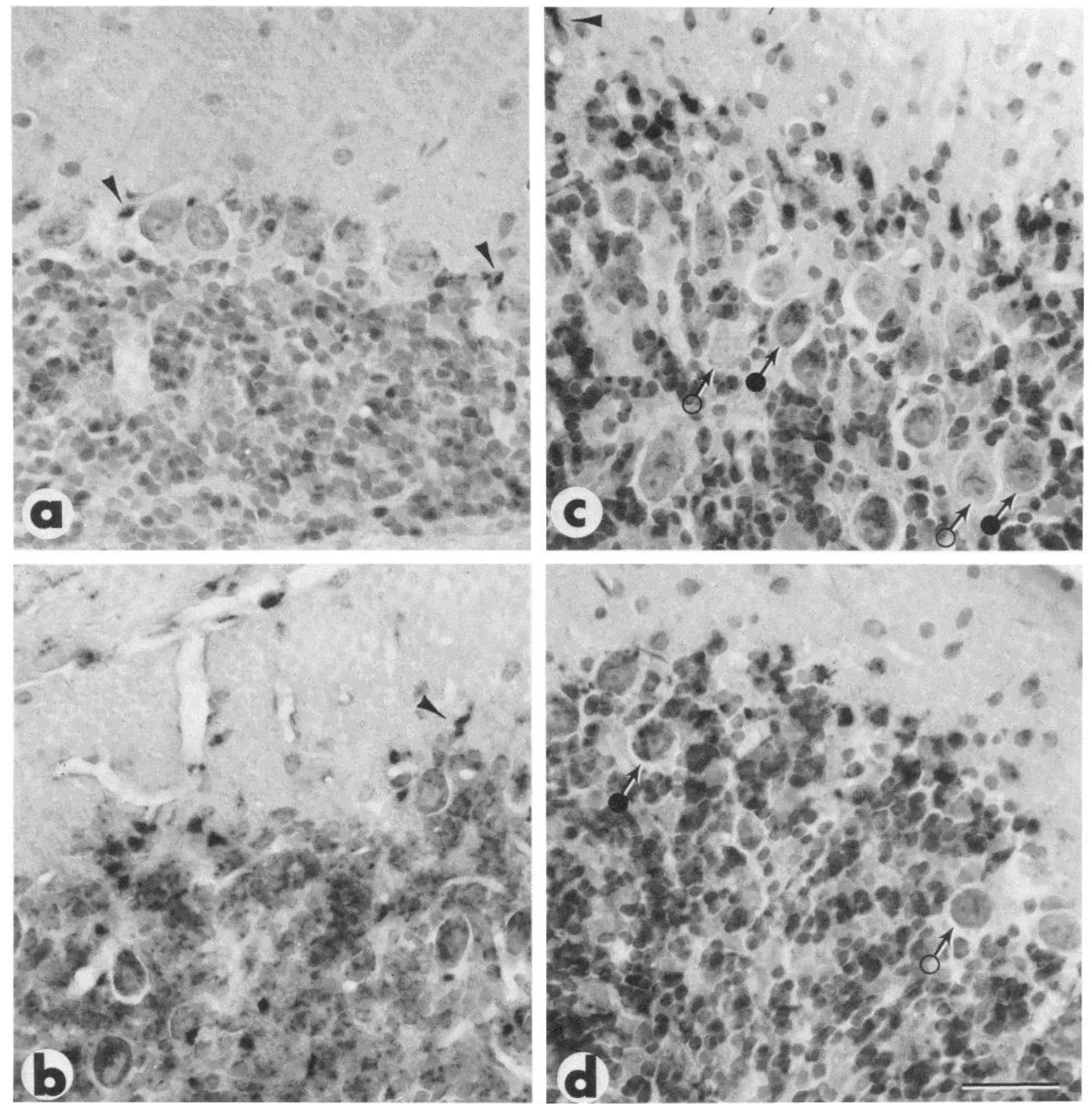

Figure 2. The granule cell, Purkinje cell, and molecular layers of $+/+, w v /+$, and $w v /-\leftrightarrow+/+$ mice from cerebella stained for $\beta$-glucuronidase activity. $a$, This section from the cerebellum of a $+/+G u s^{h} / G u s^{h}$ mouse shows the linear arrangement of the characteristically large Purkinje cells. These cells have low $\beta$-glucuronidase activity and, therefore, little or no reaction product when stained for $\beta$-glucuronidase activity. The Bergmann glia cells (arrowheads) are the only cells with staining in this neuropil. These cells are in their normal position within the Purkinje cell layer. $b$, This section from the cerebellum of a $w v /+G u s^{b} / G u s^{b}$ mouse shows the disarray in Purkinje cells that occurs in the mutant, with the large Purkinje cells scattered among the granule cells. These Purkinje cells have high $\beta$-glucuronidase activity as can be seen from the staining for the enzyme in the cell's cytoplasm. An apparently ectopic Bergmann glia cell (arrowhead) is deep in the molecular layer. $c$ and $d$, These cerebellar sections are from $w v /-G u s^{b} / G u s^{b} \leftrightarrow+/+G u s^{h} / G u s^{h}$ chimeras A and B, respectively. A nest of ectopic Purkinje cells is shown in $c$. The $\beta$-glucuronidase staining shows that these cells are derived from both the $w v /-$ and $+/+$ components of the chimera. Arrows point to some of the ectopic Purkinje cells with high (arrows with solid circles) and low (arrows with open circles) $\beta$ glucuronidase activity. An apparent Bergmann glia cell in an ectopic position is shown also (arrowhead). Two ectopic Purkinje cells, $1+/+$ and $1 \mathrm{wv} /-$, are shown in $d$. All photomicrographs were from $8-\mu \mathrm{m}$, sagittal sections. Sections were counterstained with methyl green. In $a$ through $d$, the calibration bar $=30 \mu \mathrm{m}$.

notype to the same extent as $+/+i c / i c$ (Fig. $3 c$; Table V).

It was common to find ectopic granule cells of the molecular layer occurring in small clumps. In two wv/- $+/+\leftrightarrow+/+i c / i c$ chimeras, these clumps were analyzed for the expression of the IC nuclear phenotype along with the granule cells in the granule cell layer just subjacent to the ectopic clumping of granule cells. It was thought 
TABLE V

Percentage of IC-positive granule cells in the granular and molecular layers of the cerebellum

\begin{tabular}{|c|c|c|c|c|}
\hline \multirow{2}{*}{ Genotype } & \multicolumn{2}{|c|}{ Granule Cell Layer } & \multicolumn{2}{|c|}{ Molecular Layer } \\
\hline & Individual & Mean \pm SEM & Individual & Mean \pm SEM \\
\hline & \multicolumn{2}{|c|}{$\%$} & \multicolumn{2}{|c|}{$\%$} \\
\hline \multicolumn{5}{|l|}{ Controls } \\
\hline$+/+(N=8)^{a}$ & $\begin{array}{c}6.4, \quad 7.1,3.1, \quad 4.3 \\
4.4,7.3,7.7,3.1\end{array}$ & $5.8 \pm 0.7$ & $1.7,7.8,1.4$ & $3.6 \pm 2.1$ \\
\hline$w v /+(N=3)$ & $5.2,9.5,10.1$ & $8.3 \pm 1.5$ & $3.4,2.7,11.9$ & $6.0 \pm 3.0$ \\
\hline$w v /+i c / i c(N=1)$ & 21.1 & & 26.1 & \\
\hline$i c / i c(N=7)^{a}$ & $\begin{array}{l}43.9,21.3,19.5 \\
25.5,20.9,26.0 \\
29.7\end{array}$ & $26.7 \pm 3.2$ & $\begin{array}{l}43.5,27.3,39.5 \\
\quad 31.7\end{array}$ & $35.5 \pm 3.7$ \\
\hline \multirow[t]{2}{*}{ Chimeras } & Observed & Calculated $\% \omega v /-^{b}$ & Observed & Calculated $\% w v /-^{\circ}$ \\
\hline & \multicolumn{2}{|c|}{$\%$} & \multicolumn{2}{|c|}{$\%$} \\
\hline \multicolumn{5}{|c|}{$w v /-+/+\leftrightarrow+/+i c / i c$} \\
\hline $\mathrm{C}$ & 14.9 & 56 & 3.3 & 100 \\
\hline $\mathrm{D}$ & 11.2 & 74 & 5.0 & 100 \\
\hline $\mathrm{E}$ & 11.1 & 75 & 2.3 & 100 \\
\hline $\mathrm{F}$ & 15.4 & 54 & 5.1 & 100 \\
\hline$G$ & 17.2 & 45 & 24.5 & 11 \\
\hline $\mathrm{H}$ & 27.9 & 0 & 29.0 & 0 \\
\hline I & 6.8 & 95 & 1.8 & 100 \\
\hline $\mathrm{J}$ & 1.9 & 100 & 3.6 & 100 \\
\hline
\end{tabular}

${ }^{a}$ Included in this analysis are counts of the granule cell layer from cerebella of mice from a previous study (Goldowitz and Mullen, 1982). Counts for the molecular layer granule cells (ectopic) are only from the present study.

${ }^{b}$ Calculated percentage of granule cells that are $w v /-$. The percentages are calculated as described under "Materials and Methods" using $5.8 \%$ as control score and $26.7 \%$ as the $i c$ score.

that an analysis of granule cells that arose from a similar, restricted pool of precursors might give percentages at odds with the previous, more global analysis. This was not the case. The results were the same as previously determined for whole populations of ectopic and normally positioned granule cells in these chimeras: while the granular layer had a mixture of granule cells derived from both genotypes, the molecular layer contained granule cells that did not express the IC phenotype above $+/+$ levels. A summary of our findings is presented schematically in Figure 4.

\section{Discussion}

The interpretation of data from mutant $\leftrightarrow$ normal chimeras is a process of comparing the phenotype of a cell (i.e., mutant or normal) with its genotype. If a population of chimeras always appears completely normal, it would be suggestive of a diffusible substance "curing" the disorder or of cell selection. Conversely, if the chimeras appear completely mutant, it would be suggestive of a toxic substance released from mutant cells. However, when chimeras exhibit a mosaic appearance, it suggests that the gene in question is acting within particular cells and/or specific cell interactions are involved. By using independent cell markers to determine the genotype of a cell regardless of its phenotype, it becomes possible to determine if only genotypically mutant cells appear abnormal (indicative of the gene acting within the cell) or whether genotypically wild type cells also are expressing the mutant phenotype (indicative of the gene acting in some other cell type). Of the six chimeras that clearly had a mixture of $w v /-$ and $+/+$ cells in the cerebellum, it seems most likely that the $w v /-$ component was in fact heterozygous, $w v /+$. As was implicit in our analysis of the material and presentation of the data, the chimeric cerebellar phenotypes were, in fact, intermediate to the $w v /+$ and the wild type cerebella. In light of these results, it seems reasonable to equate the tentatively used $w v /-$ designation with the $w v /+$ genotype.

Purkinje cell ectopia: A secondary effect. Several genetic mutations involving the cerebellum that affect Purkinje cells also have adverse effects on the development and/or survival of cerebellar granule cells (Purkinje cell degeneration, Ghetti et al., 1978; staggerer, Sidman, 1968; reeler, Hamburgh, 1963; and lurcher, Caddy and Biscoe, 1979). In three of these mutants, Purkinje cell degeneration (Mullen, 1977a), staggerer (Herrup and Mullen, 1979), and lurcher (Wetts and Herrup, 1982), the Purkinje cell has been shown to be a primary site of gene action though not necessarily the only one. With the exception of the mild ectopia (Rakic and Sidman, 1973b) and the slightly reduced numbers (Rezai and Yoon, 1972), there is little in $w v /+$ mice to suggesi that the Purkinje cell is a primary target of the weaver mutation. Indeed, the present study provides confirming evidence on this point at least for Purkinje cell ectopia. In the two $w v /+$ $G u s^{b} / G u s^{b} \leftrightarrow+/+G u s^{h} / G u s^{h}$ chimeras examined, Purkinje cells derived from the wild type component were as likely to be found in ectopic positions as were Purkinje cells derived from the $w v /+$ component. Thus, some factor extrinsic to the Purkinje cells is responsible for Purkinje cell ectopia in the $w v /+$ cerebellum.

Granule cells: An intrinsic site of gene action. The analysis of granule cell ectopia in weaver chimeras is 
complex. Even in normal mice, there are some ectopic granule cells, and the number varies between different strains of mice (Landis, 1973). In addition, the cell-marking system that we used (i.e., ichthyosis) requires analyz- ing a population of cells rather than identifying the genotype of individual cells with certainty. The number of IC-positive cells in the granular layer of chimera G is an example of the type of difficulties than can arise with
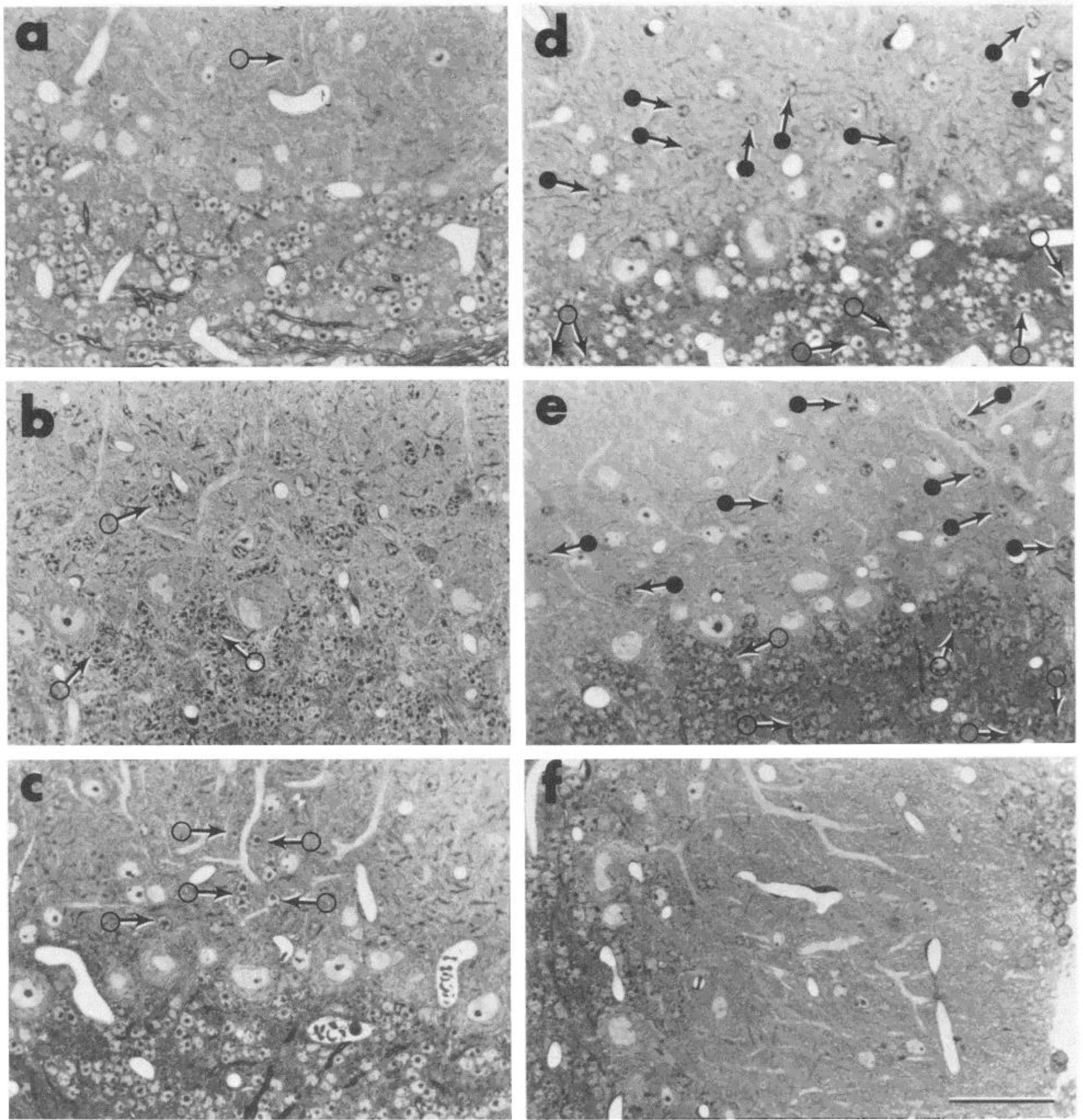

Figure 3. Toluidine blue-stained, 1- $\mu$ m sections of cerebella from $i c / i c, w v /+, w v /+i c / i c$, and $w v /-\leftrightarrow i c / i c$ mice. Arrows with open circles point to some of the IC-positive granule cells. $a$, The $i c / i c$ cerebellum has many granular layer granule cells which have the centrally clumped heterochromatin. In the molecular layer, there is an ectopic granule cell with the IC phenotype. $b$, The $w \dot{v} /+$ cerebellum has many ectopic granule cells. Arrows point to the very few granule cells of the granular layer or molecular layer (ectopic) which express the IC phenotype. $c$, Many granule cells of the $w v /+i c / i c$ double mutant cerebellum also are found in ectopic positions, and these cells frequently express the IC phenotype. $d$, Section of cerebellum from $w v /-+/+\leftrightarrow+/+i c / i c$ chimera E. None of the many ectopic granule cells (arrows with filled circles) are IC positive. In contrast to the population of molecular layer granule cells, several granule cells in the granular layer express the IC phenotype. $e$, The same point as in $d$ is illustrated here in a section from another chimeric cerebellum (chimera C). The symbols are the same as in $d$. $f$, This section is also from the cerebellum of chimera $\mathrm{C}$ and is oriented at a right angle to the fields pictured in thee other photomicrographs. A nest of ectopic granule cells is at the pial surface. None of these cells are IC positive. In $a$ through $f$, the calibration bar $=40 \mu \mathrm{m}$. 


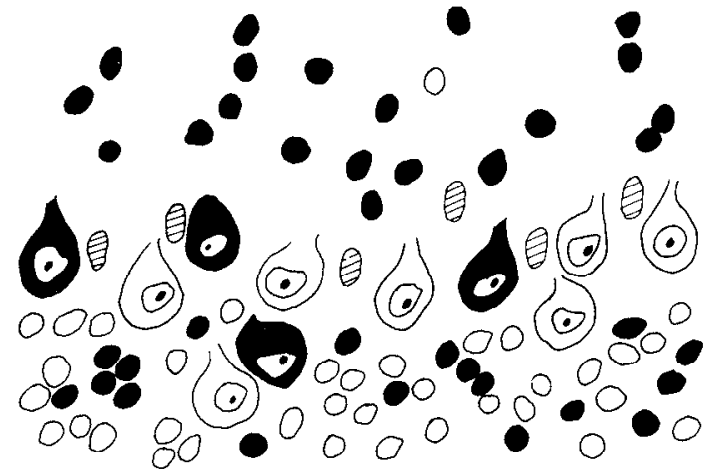

Figure 4. Schematic drawing of cells from $+/+$ (open cells) and $w v /-($ solid cells) genotypes in a chimeric $w v /-\ldots+1+$ cerebellum. The weaver phenotype of ectopic Purkinje and granule cells is shown. The ectopic Purkinje cells arise from either genetic component of the chimeric cerebellum, indicating that factors extrinsic to the Purkinje cells affect Purkinje cell ectopia in the weaver mutant cerebellum. The ectopic granule cells, however, are derived only from the weaver genetic component, indicating that the defect causing granule cell ectopia is intrinsic to this cell. The Bergmann glia cells (hatched cells) in this chimeric cerebellum are of unknown genotype.

such an analysis. Even with sampling over 500 granule cells, the moderate ic percentage of 17.4 suggested that, as calculated, $45 \%$ of the granular layer granule cells are $w v /+$ in genotype. This figure is at odds with the analysis of ectopic granule cells in a complete cerebellar section, which showed a low number of ectopic granule cells (8.4) mm of Purkinje cell layer). These differences need to be resolved by closely examining various parameters of the chimeric cerebellum and a number of chimeras. Nevertheless, after examining several chimeras, a clear pattern of results emerged.

In one chimera $(\mathrm{H})$, the cerebellum appeared completely normal and the granule cells were predominantly $+/+i c / i c$ in genotype. In two other chimeras (I and J), the cerebella were similar to $w v /+$ and the genotype analysis suggested that cells in both the granule cell layer and molecular layer were $w v /+$ in genotype (i.e., $w v /+$ $+/+)$. Since we cannot control the proportions of cells in chimeras, it is not surprising that we would find these two groups of chimeras representing opposite ends of the spectrum.

It is the third group of four chimeras that is most informative regarding the site of gene action. In these chimeras, mosaicism was evident in the granule cell layer which was a mixture of $w v /++/+$ and $+/+i c / i c$ granule cells. This was not the case, however, for the population of ectopic granule cells in the molecular layer of these four chimeras. The analysis of the heterochromatin in the ectopic granule cells indicated that no detectable population of cells had arisen from the $i c / i c$ component, and therefore, all excessive ectopic granule cells had come from the $w v /+$ component of the chimera. This could not have been due to a different phenotypic expression of heterochromatin in the nuclei of ectopic $i c / i c$ granule cells since the rare ectopic granule cells in $i c / i c$ control animals do express the peculiar nuclear phenotype that is characteristic of ic/ic granule cells in the granular layer. Furthermore, the ectopic granule cells in the cerebellum of $w v /+i c / i c$ double mutant mice also expressed the IC nuclear phenotype. Thus, in the chimeras, the observation that the ectopic granule cells are $100 \% \mathrm{wv} /++/+$ in genotype leads us to conclude that only the weaver granule cells are having difficulties migrating. Therefore, we conclude that the migratory defect in the heterozygous weaver mutant is intrinsic to the granule cell. Any other cellular site of gene action that might affect granule cell migration also would be expected to act randomly on granule cells derived from either the $w v /+$ or $i c / i c$ genotypes and produce a mixed population of ectopic granule cells. Only an unprecedented (and difficult to imagine), nearly perfect matching in the chimeric cerebellum of $w v /+$ cells and the cell type that determines successful granule cell migration could provide the results obtained in the present study. However, since granule cells arise from the external granular layer, while most other cell types arise from the ventricular zone, it would seem to be an extremely remote possibility that $w v /+$ granule cells were always juxtaposed to other cells of the same genotype. Nevertheless, this possibility could be examined in the developing cerebellum of chimeras by identifying the genetic heritage of individual granule cells and the particular cell which may serve as critical guides for granule cell migration (such as the Bergmann glia cell or the Purkinje cell; see Das et al., 1974).

The implications from the present findings are that the granule cells exert important influences upon Purkinje cell survival, positioning and form (Mariani et al., 1977), and gliomorphogenesis (see Altman, 1975) in the developing cerebellum. Previous studies (Rezai and Yoon, 1972; Rakic and Sidman, 1973b) have not found any differences between $+/+, w v /+$, and $w v / w v$ cerebella before 3 or 4 days postnatal. This suggests that the establishment of the correct number, morphology, and positioning of Purkinje and Bergmann glia cells may depend upon the granule cells' occupation of the internal granular layer for final development. Such conclusions await a closer look at the early development of the $w v /+$ and $w v /+\leftrightarrow+/+$ cerebellum. Until the causal link between gene action in the granule cell and the Purkinje and Bergmann glia cells' deficits can be established, a single site of action of the $w v$ gene and the important implications that follow are speculative.

Other possible sites of gene action and the homozygous mutant. As discussed by Mullen (1977b), when we denote a site of gene action, such as in the granule cells, we are not ruling out other sites, but these potential sites of gene action are not necessary or sufficient to create the mutant phenotype, such as the granule cell deficit in the $w v /+$ cerebellum. The cause of a reduced Purkinje cell population, Purkinje cell ectopia, aberrant Bergmann glia morphology, and ectopic Bergmann glia cells may be due to additional sites of gene action. It is well established that glial elements guide migrating cells (see Rakic, 1981). It is possible in the chimera that a pairing of $w v /+$ Bergmann glia and $w v /+$ granule cells is necessary to result in the $w v /+$ phenotype of ectopic granule cells. This possibility would lead to the prediction that we should see a dilution of the number of ectopic granule cells in chimeras since a certain percentage of $w v /+$ 
granule cells would be associated with $+/+$ Bergmann glia. However, the overall granule cell ectopia in chimeras $\mathrm{C}$ to $\mathrm{F}$ was approximately $60 \%$ of that observed in $w v /+$ mice and the calculated percentage of $w v /+$ granule cells in these chimeras was also $60 \%$ (data derived from Tables III and V). The aforementioned possibility is not, therefore, supported by the present data. Thus, the glial role may be more a matter of convenience than critical importance. Our results are interpreted not as detracting from the overall conceptual framework of glia guidance of migrating cells but as removing observations on the heterozygous weaver mutant from supporting such a role. For the $w v / w v$ cerebellum, we cannot yet make such a statement. The homozygous mutant cerebellum may present an even more complex picture. With a second dose of the weaver gene, crippling defects in additional cell types may be recruited to affect cerebellar development significantly. Our preliminary results with homozygous weaver chimeras indicate that an increased complexity of gene action may indeed be the case. Our studies on the heterozygous mutant, however, would support the hypothesis of Sotelo and Changeux (1974) that the granule cell is the primary site of gene action in the $w v / w v$ cerebellum.

Tissue culture studies designed to discriminate genetic versus environmental determinants of weaver granule cell behavior have come up with interesting results. Messer and Smith (1977) reported a cure of the $w v / w v$ granule cell deficit in a tissue culture situation. Other in vitro work, however, has found that the $w v / w v$ granule cell deficit persists (Trenkner et al., 1978; Privat et al., 1978; Willinger et al., 1981). In the study reported by Messer and Smith (1977), it may have been an artifact of the cell culture milieu, such as an arrest of further development and expression of the weaver phenotype, that enabled granule cells to survive.

Speculation on gene action in granule cells. The temporal and cytostructural localization of gene action in the granule cells is still unknown. The gene effect does not appear to be acting solely at the migratory stage of granule cell development since it has been observed that granule cells can reach the internal granule cell layer successfully before they die. The final mitotic division marks the conversion of the cell from neuroblast to differentiating neuron. This morphological and functional change must be mediated by the elaboration of a constellation of new macromolecules which the weaver gene may affect. The subsequent difficulty in migration in the $w v$ cerebellum might arise from a step in differentiation, such as axonal outgrowth (Willinger et al., 1981). Recent studies on hypo- and hyperthyroid rats suggest that granule cell fiber outgrowth may be vital to granule cell migration (Lauder, 1979). Such a role for "primitive processes" has been suggested in other developing regions of the brain (Morest, 1970). Homozygous weaver granule cells are impaired severely in elaborating any sort of axonal processes (Rakic and Sidman, 1973b). Another possibility is suggested by studies where granule cell ectopia apparently can be induced by premature contact of granule cells with nerve fibers (Ebels, 1972; Yamamoto et al., 1980; Rosenstein and Brightman, 1980). If there is genetic control on the acceptance of synapses, then in $w v /+$, this "leak" in the programmed differentiation of granule cells could account for a partial failure of migration. In the $w v / w v$ cerebellum, this presumably would be expressed as a much more incapacitating deficit leading to granule cell death. A clue to the biochemical defect in the $w v / w v$ cerebellum may have been found in the cell culture study of Trenkner et al. (1978). In culture media that had delipidated serum, the $w v$ cerebellar reaggregates had improved granule cell survival, cable formation, and granule cell migration. Further work on the temporal and spatial action of the $w v$ gene would give new information about the cellular interactions that are involved in neuronal differentiation and the elaboration of normal and mutant neuronal assemblies. Such work can provide a closer point from which to investigate the translation of genome to gene product to structural determination in the nervous system. The determination of the cellular site of gene action is a step in this process.

\section{References}

Altman, J. (1975) Postnatal development of the cerebellar cortex in the rat. IV. Spatial organization of bipolar cells, parallel fibers and glial palisades. J. Comp. Neurol. 163: 427148.

Bignami, A., and D. Dahl (1974) The development of Bergmann glia in mutant mice with cerebellar malformations: Reeler, staggerer and weaver. Immunofluorescence study with antibodies to the glial fibrillary acidic proteins. J. Comp. Neurol. 155: 219-230.

Caddy, K. W. T., and T. J. Biscoe (1979) Structural and quantitative studies on the normal $\mathrm{C} 3 \mathrm{H}$ and lurcher mutant mouse. Philos. Trans. R. Soc. Lond. (Biol.) 287: 167-201.

Caviness, V. S., Jr., and P. Rakic (1978) Mechanisms of cortical development: A view from mutations in mice. Annu. Rev. Neurosci. 1: 297-326.

Das, G. D., G. L. Camert, and J. P. McAllister (1974) Contact guidance and migratory cells in the developing cerebellum. Brain Res. 69: 13-29.

Ebels, E. J. (1972) Studies on ectopic granule cells in the cerebellar cortex-with a hypothesis as to their aetiology and pathogenesis. Acta Neuropathol. (Berl.) 21: 117-127.

Feder, N. (1976) Solitary cells and enzyme exchange in tetraparental mice. Nature 263: 67-69.

Ghetti, B., C. J. Alyea, and J. Muller (1978) Studies on the Purkinje cell degeneration (pcd) mutant: Primary pathology and transneuronal changes. J. Neuropathol. Exp. Neurol. 37: 617.

Goldowitz, D., and R. J. Mullen (1982) Nuclear morphology of ichthyosis mutant mice as a cell marker in chimeric mice. Dev. Biol. 89: 261-267.

Hamburgh, M. (1963) Analysis of the postnatal developmental effects of "recler," a neurological mutation in mice. A study in developmental genetics. Dev. Biol. 8: 165-185.

Hayashi, M., T. Nakajima, and W. H. Fishman (1964) Distribution of $\beta$-glucuronidase activity in rat tissues employing the naphthol AS-BI glucuronide hexazonium pararosanalin method. J. Histochem. Cytochem. 12: 293-297.

Herrup, K., and R. J. Mullen (1979) Staggerer chimeras: Intrinsic nature of Purkinje defects and implications for normal cerebellar development. Brain Res. 178: 443-457.

Hirano, A., and H. M. Dembitzer (1973) Cerebellar alterations in the weaver mouse. J. Cell Biol. 56: 478-486.

Hirano, A., H. M. Dembitzer, and C. H. Yoon (1977) Development of Purkinje cell somatic spines in weaver mouse. Acta Neuropathol. (Berl.) 40: 85-90.

Karnovsky, M. J. (1965) A formaldehyde-glutaraldehyde fixa- 
tive of high osmolality for uses in electron microscopy. J. Cell Biol. 27: 137A-138A.

Landis, S. C. (1973) Granule cell heterotopia in normal and nervous mutant mice of the BALB/c strain. Brain Res. 61: 175-189.

Lauder, J. M. (1979) Granule cell migration in developing rat cerebellum. Influence of neonatal hypo- and hyperthyroidism. Dev. Biol. 70: 105-115.

Messer, A., and D. M. Smith (1977) In vitro behavior of granule cells from staggerer and weaver mutants of mice. Brain Res. 130: $13-23$.

Morest, D. K. (1970) A study of neurogenesis in the forebrain of opossum pouch young. Z. Anat. Entwicklungsgesch. 130: 265-305.

Mugnaini, E., and P. F. Forstrønen (1967) Ultrastructural studies on cerebellar histogencsis. I. Differentiation of granule cells and development of glomeruli in the chick embryo. Z. Zellforsch. Mikrosk. Anat. 77: 115-143.

Mullen, R. J. (1977a) Site of pcd gene action and Purkinje cell mosaicism in cerebella of chimaeric mice. Nature 270: 245247.

Mullen, R. J. (1977b) Genetic dissection of the CNS: Mutant $\leftrightarrow$ normal mouse and rat chimeras. In Society for Neuroscience Symposium. Vol. 2: Approaches to the Cell Biology of Neurons, W. M. Cowan and J. A. Ferrendelli, eds., Society for Neuroscience, Bethesda, MD.

Mullen, R. J., and K. Herrup (1979) Chimeric analysis of mouse cerebellar mutants. In Neurogenetics: Genetic Approaches to the Nervous System, X. O. Breakefield, ed., pp. 173-196, Elsevier, New York.

Mullen, R. J., and W. K. Whitten (1971) Relationship of genotype and degree of chimerism in coat color to sex ratios and gametogenesis in chimeric mice. J. Exp. Zool. 178: 165-176.

Privat, A., A. M. Marson, and M. J. Drian (1978) In vitro models of neural growth and differentiation. Prog. Brain Res. 51: $335-356$.

Rakic, P. (1971) Neuron-glia relationships during granule cell migration in developing cerebellar cortex. A Golgi and electron microscopic study in Macacus rhesus. J. Comp. Neurol. 141: 283-312.

Rakic, P. (1981) Neuronal-glial interaction during brain development. Trends Neurosci. 4: 184-187.

Rakic, P., and R. L. Sidman (1973a) Weaver mutant mouse cerebellum: Defective neuronal migration secondary to abnormality of Bergmann glia. Proc. Natl. Acad. Sci. U. S. A. 70: $240-244$.
Rakic, P., and R. L. Sidman (1973b) Sequence of developmental abnormalities leading to granule cell deficit in cerebellar cortex of weaver mutant mice. J. Comp. Neurol. 152: 103132.

Rakic, P., and R. L. Sidman (1973c) Organization of cerebellar cortex secondary to deficit of granule cell in weaver mutant mice. J. Comp. Neurol. 152: 133-162.

Rezai, Z., and C. H. Yoon (1972) Abnormal rate of granule cell migration in the cerebellum of "weaver" mutant mice. Dev. Biol. 29: 17-26.

Rosenstein, J. M., and M. W. Brightman (1980) Arrest and migration of cerebellar neurons towards grafts of autonomic ganglion. Peptides Suppl. 1 1: 221-227.

Sidman, R. L. (1968) Development of interneuronal connections in brains of mutant mice. In Physiological and Biochemical Aspects of Nervous Integration, F. D. Carlson, ed., pp. 163193, Prentice Hall, Englewood Cliffs, NJ.

Sidman, R. L., J. B. Angevine, and E. Taber Pierce (1971) Atlas of the Mouse Brain and Spinal Cord, Harvard University Press, Cambridge, MA.

Sotelo, C. (1975) Anatomical, physiological and biochemical studies of the cerebellum from mutant mice. II. Morphological study of cerebellar cortical neurons and circuits in the weaver mouse. Brain Res. 94: 19-44.

Sotelo, C. (1980) Mutant mice and the formation of cerebellar circuitry. Trends Neurosci. 3: 33-36.

Sotelo, C., and J. P. Changeux (1974) Bergmann fibers and granule cell migration in the cerebellum of homozygous weaver mutant mouse. Brain Res. 77: 484-491.

Swarz, J., and M. Oster-Granite (1978) Presence of radial glia in foetal mouse cerebellum. J. Neurocytol. 7: 301-312.

Trenkner, E., M. E. Hatten, and R. L. Sidman (1978) Effect of ether-soluble serum components in vitro on the behavior of immature cerebellar cells in weaver mulant mice. Neuroscience 3: 1093-1100.

Wetts, R., and K. Herrup (1982) Interaction of granule, Purkinje and inferior olivary neurons in Lurcher chimaeric mice. I. Qualitative studies. J. Embryol. Exp. Morphol., 68: 87-98.

Willinger, M., D. M. Margolis, and R. L. Sidman (1981) Neuronal differentiation in cultures of weaver $(w v)$ mutant mouse cerebellum. J. Supramol. Struct. 17: 79-86.

Yamamoto, M., V. Chan-Palay, H. W. M. Steinbusch, and S. L. Palay (1980) Hyperinnervation of arrested granule cells produced by the transplantation of monoamine-containing neurons into the fourth ventricle of rat. Anat. Embryol. (Berl.) 159: $1-15$. 